Kaum eines der vielen auf den Bildungsmarkt drängenden Bücher kann angesichts dieser Umstände allein die ganze Komplexität des europäischen Einigungsprozesses erfassen. Es ist das Verdienst der neuen Veröffentlichung von Winfried Loth, eine Brücke über all jene Flüsse und Bäche (gelegentlich ist es auch nur ein Rinnsal) zu spannen, aus denen unterdessen die europawissenschaftliche Literatur anschwillt $\mathrm{zu}$ einem großen, aber oft undurchdringlichen Literatur-See.

Erfreulich ist die durchgängig „europäische" Sicht der Studie. Nicht immer frei von soupcons bleiben die Einlassungen Loths zum europäisch-amerikanischen Verhältnis. Dabei gehört die amerikanische Unterstützung zum Gründungprozess der EWG ebenso wie der Beitrag der USA zur Befreiung Europas von Hitlers Barbarei. Und das nächste Kapitel der europäischen Einigung dürfte die Frage nach der $\mathrm{Zu}$ kunftsfähigkeit der Atlantischen Zivilisation, von der die EU die eine Säule ist, auf ganz neue Weise behandeln: TTIP-Verhandlungen im Schatten des Putinismus - so könnte schon bald ein weiteres Kapitel in einer wünschenswerten erweiterten Neuauflage von Wilfrieds Loths Meisterwerk überschrieben sein.

Ludger Kühnhardt

\section{POLITISCHE BILDUNG}

Fritz Borinski: The German Volkshochschule. An Experiment in Democratic Adult Education under the Weimar Republic. Herausgegeben, eingeleitet und mit Annotationen und einem prosopographischen Anhang versehen von Martha Friedenthal-Haase, (Beiträge zur internationalen, interkulturellen und historischen Erwachsenenbildung) Bad Heilbronn: Julius Klinkhardt 2014, 285 S., 18,90 €.

Die Gründungsphase unseres Faches nach 1945 zeichnete sich durch eine vielfältige Verbindung zwischen der akademischen Wissenschaft von der Politik und der praktischen politischen Bildungsarbeit aus. Ganz allgemein verstand sich das Fach als eine Demokratiewissenschaft, die sich durch ihren zentralen Beitrag zum Aufbau einer staatsbürgerlichen Kultur im neuen Deutschland legitimierte. Im Besonderen fanden viele Studierenden der Politikwissenschaft ihren Beruf in der Erwachsenenbildung. Das trifft vor allem auf die Absolventen der Westberliner Deutschen Hochschule für Politik und ihrer Nachfolgeinstitution an der Freien Universität, des Otto-Suhr-Instituts, zu. Und schließlich verdankte die junge Disziplin ihre spektakuläre Expansion und dauerhafte Etablierung der Verankerung in der Lehrerausbildung für das neue Schulfach der Gemeinschaftsoder Sozialkunde, das ab dem Ende der 1950er Jahre an den bundesdeutschen Schulen eingeführt wurde.

Diese Symbiose von Politikwissenschaft und politischer Bildung, insbesondere der Erwachsenenbildung, hat schon Hermann Heller verkörpert, eine zentrale, wenn auch zu früh (1933) verstorbene Gründungsfigur der bun- 
desdeutschen Politikwissenschaft. Dieser prominente sozialdemokratische Staatsrechtslehrer der Weimarer Republik hatte in seinen letzten Lebensjahren eine „Staatslehre“ verfasst, die nach ihrer posthumen Publikation im Jahr 1935 und zahlreichen Neuauflagen in der jungen Bundesrepublik zum programmatischen Grundlagenwerk der deutschen Politikwissenschaft wurde. Gleichzeitig war Heller, vor allem während seiner Zeit Anfang der 1920er Jahre an der Leipziger Universität, Protagonist der demokratischen Volkshochschulbewegung und insbesondere der Bemühungen auf dem Gebiet der Arbeiterbildung.

Wie kein anderer hat der Heller-Schüler Fritz Borinski (1903-1988) diese Verknüpfung von akademischem Fach und politischer Bildungsarbeit repräsentiert. Dieser protestantische Spross aus einer Familie des assimilierten Berliner Judentums hat sein juristisches Hauptstudium 1927 mit einer Dissertation über „Joseph Görres und die deutsche Parteienbildung “ abgeschlossen, nach heutigen Kriterien eine politikwissenschaftliche Arbeit. Schon während des Studiums fand Borinski seinen Beruf in der Erwachsenbildung, übernahm schließlich 1931 als Assistent des Philosophen und Pädagogen Theodor Litt die Leitung des „Seminars für freies Volksbildungswesen " an der Leipziger Universität. 1934 aus dieser Stellung aus politischen und rassistischen Gründen entlassen, emigrierte Borinski nach England.

Auf Ermunterung des an der London School of Economics lehrenden Soziologen Karl Mannheim verfasste Borinski 1944/45 ein Manuskript über die deutsche Volkshochschulbewegung in der Weimarer Republik, ihre historische Entwicklung, ihre gesellschaftlichen, po- litischen und pädagogischen Herausforderungen und nicht zuletzt über die Lehren für den Aufbau einer zweiten, stabileren deutschen Demokratie. Dieser Text ist nun nach langen Jahrzehnten von Martha Friedenthal-Haase, einer emeritierten Jenenser Professorin der Erwachsenenbildung, veröffentlicht worden. Er ist ein weiteres überzeugendes Beispiel dafür, dass die in der Zeit der Emigration verfassten Texte eine geistige Brücke zwischen der ersten und der zweiten deutschen Demokratie bilden - eine Einsicht, die in unserem Fach bereits durch die Arbeiten von Alfons Söllner eröffnet worden ist.

Im Mittelpunkt des Buches steht die Darstellung der Organisation, der Inhalte und der Probleme der unterschiedlichen Institutionen der Volkshochschulbewegung zur Zeit der Weimarer Republik, mit den Abendvolkshochschulen und den Heimvolkshochschulen an der Spitze. Es wird deutlich, dass die Initiativen zu dieser Bewegung nach dem Ersten Weltkrieg von den Bemühungen zu einer Demokratiereform ausgingen, darin nicht unähnlich dem zeitgeschichtlichen Hintergrund der 1920 gegründeten Deutschen Hochschule für Politik. Auf der anderen Seite führte die 1929 einsetzende wirtschaftliche, gesellschaftliche und politische Krise der Weimarer Demokratie auch zum Untergang der freien Volkshochschulbewegung.

Doch Borinski beließ es nicht bei diesem vom persönlichen Miterleben geprägten historischen Bericht, er formulierte in seinem abschließenden Kapitel auch die Zukunftsaufgaben der Volkshochschulbewegung und allgemein der politischen Bildungsarbeit beim zweiten Anlauf einer Demokratiegründung in Deutschland, der aus dem Scheitern des ersten Versuchs lernen konnte. 
Dazu gehörte für ihn an erster Stelle die Einsicht, dass eine demokratische Ordnung sich nicht auf die formalen Strukturen der staatlichen Ordnung beschränken soll, sondern auf einer politische Kultur der staatsbürgerlichen Verantwortung und einer Gesellschaftspolitik der ,integralen Demokratie" aufzubauen hat.

Fritz Borinski hat dieses in seiner Exilzeit entwickelte Programm eines Neuanfangs der politischen Bildung nach seiner Rückkehr nach Deutschland im Jahr 1947 persönlich umgesetzt. Er war zunächst Leiter einer niedersächsischen Heimvolkshochschule und dann der großen Bremer Volkshochschule. Nachdem er 1954 in einem programmatischen Buch über den „Weg zum Mitbürger“ die „politische Aufgabe der freien Erwachsenenbildung in Deutschland" - so der Untertitel - konzipiert hatte, wurde Borinski zwei Jahre später auf einen Lehrstuhl an der Freien Universität in Berlin berufen. Dort hat er in den 15 Jahren bis zu seiner Emeritierung nicht nur das pädagogische Teilgebiet der Erwachsenenbildung in Lehre und Forschung aufgebaut, sondern ein ganzes Spektrum von Initiativen zur politischen Bildungsarbeit der Universität entwickelt, oft in Kooperation mit Professorenkollegen am Otto-SuhrInstitut, noch mehr unter Einbeziehung von jungen Studenten und Doktoranden der Politikwissenschaft wie dem Verfasser dieser Rezension.

Die durch die Studentenbewegung ausgelöste Vertrauenskrise hat auch die Arbeit dieses Pioniers der deutschen Erwachsenenbildung in Mitleidenschaft gezogen. Doch seine Maxime, dass eine stabile Demokratie die politische Bildungsarbeit benötige wie umgekehrt eine staatsbürgerliche Erziehung nur in der Demokratie möglich sei, gilt auch heute noch. Umso bedauerlicher erscheint es, dass heute in der deutschen Politikwissenschaft deren Verbindungen zu Praxis und Theorie der politischen Bildung, von Ausnahmen angesehen, weitgehend verblasst sind. Die Lektüre dieser von der Herausgeberin hervorragend edierten Exilschrift aus dem Jahre 1944/45 ist wieder einmal ein Beispiel dafür, welche Schätze noch für unsere Gegenwartsbestimmung und Zukunftsorientierung aus der Vergangenheit zu heben sind.

Wilhelm Bleek 